\title{
Elevated ALS Biomarker Levels in CSF of a FTD Patient at the Presymptomatic Stage of ALS
}

\author{
Emmanuel Cognat, *† Maxim De Schaepdryver, $\neq$ Jacques Hugon, $* \$$ \\ Koen Poesen, $\neq$ and Claire Paquet $* \dagger$
}

Key Words: frontotemporal lobar degeneration, amyotrophic lateral sclerosis, biomarker, neurofilament, prognosis, cerebrospinal fluid

(Alzheimer Dis Assoc Disord 2017;00:000-000)

$F_{\mathrm{d} e}^{\mathrm{r}}$ rontotemporal lobar degeneration (FTLD) is a neurodegenerative disease associated with amyotrophic lateral sclerosis (ALS). ${ }^{1}$ Indeed, prospective studies have shown that $12.5 \%$ to $14 \%$ of patients with FTLD have also ALS. ${ }^{2,3}$

Neurofilaments are intermediate filaments of neuronal cytoskeleton that are sensitive markers of axonal damage and neurodegeneration. ${ }^{4}$ Neurofilament heavy chain $(\mathrm{pNfH})$ and neurofilament light chain (NfL) in cerebrospinal fluid (CSF) have been proposed as diagnostic and prognostic biomarkers of clinical ALS. ${ }^{5-7}$ Increased CSF NfL levels have been reported in FTLD to reflect disease severity. ${ }^{8}$ To date, no biomarker has been reported to detect presymptomatic ALS in FTLD patients. We report a case of a FTLD patient with elevated CSF pNFH more than 1 year before the onset of ALS, suggesting that $\mathrm{pNfH}$ might be a biomarker of presymptomatic ALS in FTLD patients.

\section{CASE REPORT}

A 56-year-old man was referred in June 2013 to our memory center for behavioral changes and cognitive complaints. Familial history included cases of alcoholism (mother, 2 maternal uncles, 1 brother) and a maternal premature death. In 2011, an episode of depression secondarily associated with delusional jealousy was noted. $\mathrm{He}$ subsequently developed aggressive behavior, rudeness, disinhibition, and cognitive dysfunction that progressed over time. Neurological examination showed normal motor function as well as the absence of fasciculations, amyotrophy and pyramidal syndrome, as repeatedly observed independently since 2011 by 3 different neurologists. Neuropsychological evaluation showed severe executive dysfunction with impaired working and episodic memories and preserved instrumental functions. Brain magnetic resonance imaging and biological analyses, including standard CSF analysis, were normal. CSF amyloid $(\mathrm{A} \beta)_{1-42},(\mathrm{~A} \beta)_{1-40}$, their ratio and phosphorylated

Received for publication July 28, 2017; accepted September 27, 2017. From the *Cognitive Neurology Center AP-HP, GH Saint-Louis Lariboisière Fernand-Widal; †INSERM, U942; §University Paris Diderot, Sorbonne Paris Cité, Paris, France; and $\ddagger$ Laboratory for Molecular Neurobiomarker Research, Department of Neurosciences, KU Leuven (University of Leuven) and Laboratory Medicine, University Hospitals Leuven, Leuven, Belgium.

K.P. and C.P. contributed equally to the work.

E.C.: wrote the first draft of the manuscript. K.P. and C.P.: directed the work and supervised the writing. C.P.: involved in patient care.

The authors declare no conflicts of interest.

Reprints: Claire Paquet, Cognitive Neurology Center, GH Saint-Louis Lariboisière Fernand-Widal, APHP, 200 rue du Faubourg Saint-Denis, Paris 75010, France (e-mail: claire.paquet@inserm.fr).

Copyright (C) 2017 Wolters Kluwer Health, Inc. All rights reserved. tubulin-associated units were normal while total tubulin-associated units was slightly increased. CSF NfL and pNfH levels were increased with respect to the reference value to distinguish ALS from non-MND diseases (Table 1). Serum progranulin level was normal Genetic testing for C9ORF72 and MAPT mutations were negative. According to the revised International Behavioural Variant FTLD Consortium criteria, a diagnosis of behavioral variant FTLD was made.

During the summer of 2014, the patient complained of bilateral hand clumsiness, which progressively worsened during the following weeks. Neurological examination on September 2014 revealed diffuse fasciculations, a pyramidal syndrome and distal bilateral upper limb motor deficit. Furthermore, patient's relatives described occasional abnormal deglutition. Cervical spine magnetic resonance imaging ruled out local spinal cord compression and electroneuromyography (ENMG) confirmed probable ALS associated with behavioral variant FTLD according to the revised El Escorial criteria. Behavioral, cognitive, and motor deficits worsened and the patient died in December 2014 of a severe lung infection.

\section{DISCUSSION}

Up to $36 \%$ of FTLD patients without ALS demonstrate clinical evidence of minor motor system dysfunction. ${ }^{2,3} \mathrm{Sim}-$ ilarly, Burrell et al ${ }^{3}$ identified neurophysiological signs of upper (reduced short-interval intracortical inhibition) and lower (reduced neurophysiological index) motor neuron dysfunction in FTLD patients. However, the significance of these findings is uncertain and there is currently no clinical or electrophysiological predictor of upcoming ALS development.

Increased CSF levels of both pNfH and NfL have been well documented in clinical ALS (with or without FTLD).${ }^{5,6,9}$ In contrast, elevated CSF NfL levels, ${ }^{8}$ but not $\mathrm{pNfH},{ }^{9}$ have been reported in FTLD. Indeed, we have recently demonstrated that $\mathrm{pNfH}$ discriminated between ALS and disease controls including patients with FTLD. The majority of FTLD patients had pNfH levels well below or at about the cutoff of $618 \mathrm{pg} / \mathrm{mL}$, although 2 patients out of 46 patients with FTLD had pNfH levels in CSF in the range of our case (ie, 947 and $1633 \mathrm{pg} / \mathrm{mL}$, the former suffering from corticobasal syndrome with pyramidal signs on right side of the body, the latter without clinical complaints of motor dysfunction to date). ${ }^{7}$ Another study measured levels in CSF $>618 \mathrm{pg} / \mathrm{mL}$ in about 5 of 17 patients with FTLD. ${ }^{10}$ The latter employed, however, a different assay (termed hyperphosphorylated $\mathrm{NfH}$ or $\mathrm{NfH}^{\mathrm{SMI} 34}$ ) demonstrated to generate higher values as compared with other assays for phosphorylated $\mathrm{pNfH} .{ }^{11}$ Hence, values between different assays are not commutable, which hinders a comparison among studies.

Several limitations can be put forward to this case report. First, due to the opposition of the patient, the ENMG was lacking at the moment of the CSF biomarkers analysis. The motor neurological examinations were normal when FTLD was initially diagnosed but we cannot rule out 
TABLE 1. CSF Biomarkers Results at June 12, 2013

\begin{tabular}{|c|c|c|c|}
\hline Biomarker in CSF & Method (Catalog Number) & $\begin{array}{c}\text { Value } \\
(\mathrm{pg} / \mathrm{mL})\end{array}$ & $\begin{array}{c}\text { Reference } \\
\text { Value }(\mathrm{pg} / \mathrm{mL}) \\
\end{array}$ \\
\hline $\mathrm{A} \beta_{1-42}$ & Innotest ELISA kit (\#81576) & 1189 & $>815$ \\
\hline $\mathrm{A} \beta_{1-40}$ & Innotest ELISA kit (\#80462) & 14,937 & $2000<\mathrm{N}<20,000$ \\
\hline $\mathrm{A} \beta_{1-42 / 1-40}$ ratio & & 0.08 & $>0.05$ \\
\hline Total tau & Innotest ELISA kit (\#81572) & 328 & $<300$ \\
\hline Phosphorylated tau $(181 \mathrm{P})$ & Innotest ELISA kit (\#81574) & 48 & $<58$ \\
\hline Neurofilament light chain (NfL) & Uman Diagnostics ELISA kit (\#10-7001) & 12,394 & $<3819^{7}$ \\
\hline Phosphorylated neurofilament heavy chain ( $\mathrm{pNfH}$ ) & Biovendor ELISA kit (\#RD191138300R) & 1548 & $<618^{7}$ \\
\hline
\end{tabular}

CSF indicates cerebrospinal fluid; tau, tubulin-associated units.

that minor ENMG abnormalities could have been detected at the presymptomatic phase of the ALS. Second, due to possible family history without identified genetic abnormality, we cannot exclude that ALS-FTLD observed in this patient is linked to a TARDBP or SOD1 mutation or another not yet detected origin that might prevent our findings from generalization to all ALS-FTLD patients.

In conclusion, we show increased CSF pNfH levels in a FTLD patient more than 1 year before the onset of clinical ALS. Our observation warrants further research for $\mathrm{pNfH}$ as a prognostic marker for motor neuron disease in FTLD patients, as has been previously proposed. ${ }^{9,11}$

\section{ACKNOWLEDGMENT}

The authors would like to thank Pr Jean-Louis Laplanche and Dr Elodie Amar for their help with AD CSF biomarkers.

\section{REFERENCES}

1. Mackenzie IRA, Feldman HH. Ubiquitin immunohistochemistry suggests classic motor neuron disease, motor neuron disease with dementia, and frontotemporal dementia of the motor neuron disease type represent a clinicopathologic spectrum. J Neuropathol Exp Neurol. 2005;64:730-739.

2. Lomen-Hoerth C, Anderson T, Miller B. The overlap of amyotrophic lateral sclerosis and frontotemporal dementia. Neurology. 2002;59:1077-1079.
3. Burrell JR, Kiernan MC, Vucic S, et al. Motor neuron dysfunction in frontotemporal dementia. Brain. 2011;134:2582-2594.

4. Petzold A. Neurofilament phosphoforms: surrogate markers for axonal injury, degeneration and loss. J Neurol Sci. 2005;233: $183-198$.

5. Oeckl P, Jardel C, Salachas F, et al. Multicenter validation of CSF neurofilaments as diagnostic biomarkers for ALS Amyotroph Lateral Scler Front Degener. 2016;17:404-413.

6. Gendron TF, C9ORF72 Neurofilament Study Group, Daughrity LM, et al. Phosphorylated neurofilament heavy chain: a biomarker of survival for C9ORF72-associated amyotrophic lateral sclerosis. Ann Neurol. 2017;82:139-146.

7. Poesen K, De Schaepdryver M, Stubendorff B, et al. Neurofilament markers for ALS correlate with extent of upper and lower motor neuron disease. Neurology. 2017;88:1-8.

8. Scherling CS, Hall T, Berisha F, et al. Cerebrospinal fluid neurofilament concentration reflects disease severity in frontotemporal degeneration. Ann Neurol. 2014;75: 116-126.

9. Weydt P, Oeckl P, Huss A, et al. Neurofilament levels as biomarkers in asymptomatic and symptomatic familial amyotrophic lateral sclerosis. Ann Neurol. 2016;79:152-158.

10. Pijnenburg YAL, Janssen JC, Schoonenboom NSM, et al. CSF neurofilaments in frontotemporal dementia compared with early onset Alzheimer's disease and controls. Dement Geriatr Cogn Disord. 2007;23:225-230.

11. Ganesalingam J, An J, Shaw CE, et al. Combination of neurofilament heavy chain and complement c3 as CSF biomarkers for ALS. J Neurochem. 2011;117:528-537. 